\title{
DFT Description of Intermolecular Forces between 9-Aminoacridines and DNA Base Pairs
}

\author{
Sandra Cotes Oyaga, ${ }^{1}$ José Cotuá Valdés, ${ }^{2}$ Sigrid Borja Paez, ${ }^{3}$ and Keylin Hurtado Marquez ${ }^{3}$ \\ ${ }^{1}$ Departamento de Química y Biología, Universidad del Norte, Km 5 Antigua vía a Puerto Colombia, Barranquilla, Colombia \\ ${ }^{2}$ Grupo de Investigación Max Planck, Universidad del Atlántico, Km 7 Antigua vía a Puerto Colombia, Barranquilla, Colombia \\ ${ }^{3}$ Universidad del Atlántico, Km 7 Antigua vía a Puerto Colombia, Barranquilla, Colombia \\ Correspondence should be addressed to Sandra Cotes Oyaga; scotes@uninorte.edu.co
}

Received 15 March 2013; Revised 29 May 2013; Accepted 16 July 2013

Academic Editors: A. M. Lamsabhi, G. Monard, and H.-Y. Zhang

Copyright (c) 2013 Sandra Cotes Oyaga et al. This is an open access article distributed under the Creative Commons Attribution License, which permits unrestricted use, distribution, and reproduction in any medium, provided the original work is properly cited.

\begin{abstract}
The B3LYP method with 6-31G* basis set was used to predict the geometries of five 9-aminoacridines (9-AA 1(a-e)), DNA base pairs, and respective complexes. Polarizabilities, charge distribution, frontier molecular orbital (FMO), and dipole moments were used to analyze the nature of interactions that allow reasonable drug diffusion levels. The results showed that charge delocalization, high polarizabilities, and high dipole moments play an important role in intermolecular interactions with DNA. The interactions of 9-AA 1(a-e) with GC are the strongest. 9-AA 1(d) displayed the strongest interaction and 9-AA 1(b) the weakest.
\end{abstract}

\section{Introduction}

Intermolecular recognition is a key process for the interaction with biological systems. The strength of intermolecular weak interactions such as hydrogen bonding, Van der Waals forces, aromatic stacking, binding, and intercalation is closely related to biological activity. Acridines are known as antitumor [15], antiviral [6-8], antiprion [9, 10], antimicrobial [11], antiinflammatory, and analgesic [12].

9-Aminoacridines (9-AA) have also been considered for the treatment of protozoal infections [13], and anticancer activity was first considered in the 1940s [14]. Since then, a large number of 9-aminoacridine drugs, natural alkaloids, or synthetic molecules have been tested as antitumoral agents. 9AA activity is due to the intercalation of the tricyclic aromatic ring between adjacent base-pairs [15]. Thereby high affinity for DNA is the result of stacking between base-pairs and enhanced by electrostatic and hydrophobic interactions with other substituents. Strong binding correlates cytotoxicity; however, extravascular distribution is limited by low levels of circulating free drug. Thus biological activity is the result of a compromise between binding and circulating drug levels.

Sebestik et al. [16, 17] reported the synthesis of acridine conjugates 9-AA $\mathbf{1}(\mathbf{a}-\mathbf{e})$ possessing amino acids bonded to an ethylenediamine linker that showed low affinity to nucleic acids. Sebestik et al. reduced the DNA-binding constant of 9-aminoacridines by introduction of DNA binders with different base-pair affinity. 9-AA as a GC-DNA binder and peptide residues as AT-DNA binders were connected to the ethylenediamine linker. The peptide conjugates 9-AA $\mathbf{1}(\mathbf{a}-\mathbf{e})$ showed weak interactions with DNA due to the competitive role of substituents.

DFT methods are accurate methods for computational studies [18, 19], and electrostatic interactions are described by available force fields [20-22]. However, the quest for DFT methods which accurately describe long-range electron correlation continues to be a challenge in computational chemistry. Modeling of DNA interactions with 9-AA offers an insight about the physical and chemical phenomena that are not measurable through experimentation. In this work, the structures and electrostatic interactions of 9-AA $1(\mathbf{a}-$ e) with AT and GC (Figure 1) are calculated and analyzed. To our knowledge, this is the first report showing the predicted electrostatic interactions of these molecules with DNA base-pairs that might be responsible for the observed weak interactions. The effect of polarizability, dipole moment, FMO energies of the base-pair with 9-AA $\mathbf{1}(\mathbf{a}-\mathbf{e})$ was also studied by DFT. Further improvements in the quantum 


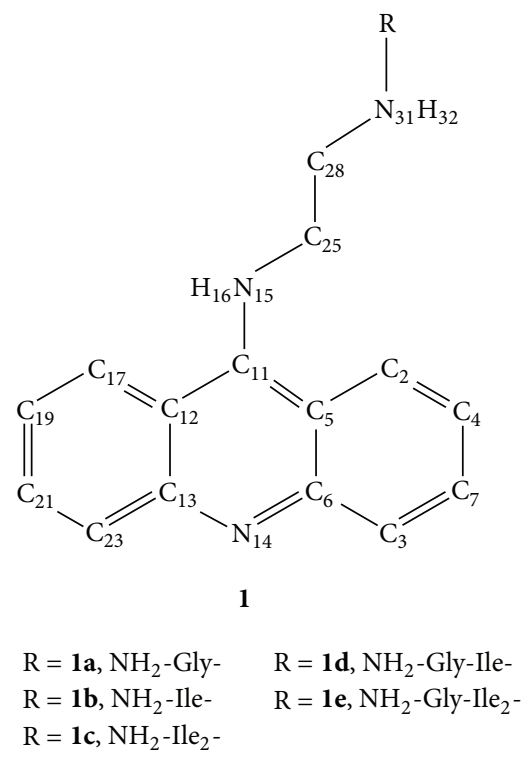

FIGURE 1: Substituted 9-aminoacridines 1(a-e).

TABLE 1: Energies of optimized 9-AA 1(a-e)-base-pair complexes calculated at the B3LYP/6-31G* basis set.

\begin{tabular}{lcc}
\hline 9-AA & $\begin{array}{r}\text { AT complex } \\
\text { Energy (au) }\end{array}$ & $\begin{array}{r}\text { GC complex } \\
\text { Energy (au) }\end{array}$ \\
\hline $\mathbf{1 ( a )}$ & 1874,420 & 1890,414 \\
$\mathbf{1 ( b )}$ & 2031,520 & 2047,671 \\
$\mathbf{1}(\mathbf{c})$ & 2396,975 & 2412,918 \\
$\mathbf{1 ( d )}$ & 2239,640 & 2255,649 \\
$\mathbf{1 ( e )}$ & 2604,928 & 2620,917 \\
\hline
\end{tabular}

chemistry calculations of base-pair interactions with 9-AA $\mathbf{1}(\mathbf{a}-\mathbf{e})$ including intermolecular electron correlation effects remain to be done as a continuing effort to estimate basepairing energies sufficiently accurate for most applications.

\section{Computational Methods}

Calculations on the isolated molecules and molecular complexes were performed using Spartan '08 [18] molecular mechanic MMFF method. Geometries optimizations were performed at the B3LYP level using $6-31 G^{*}$ basis set. All geometries correspond to potential energy minima as shown by harmonic normal-mode analysis at the same level of theory. Molecular descriptors such as dipole moment, polarizability, and FMO calculations have been carried out using the B3LYP/6-31G** optimized geometries within Gaussian 09W [23].

\section{Results and Discussion}

Numbering of the DNA base-pairs is shown in Figure 2. Optimized structures of the molecular complexes between 9-AA $\mathbf{1}(\mathbf{a}-\mathbf{e})$ and base-pairs are shown in Figures 3 and 4. Equilibrium geometries energetics are shown in Table 1. From equilibrium geometries shown in Figures 3 and 4, it is seen that coplanarity is not retained in the complex of 9-AA $\mathbf{1 ( c )}$ with AT and complexes with GC.

Atomic charges, polarizability, dipole moment, and FMO are shown in Tables 2-4. Among the atoms of the heterocyclic ring in the isolated 9-AA 1(a-e), C13 and C6 have the maximum positive charges which are caused by the bonding to a negatively charged electronegative N14. Instead 9-AA 1(c) exhibits a maximum positive charge in C11, which is bonded to a negatively charged N15. See Table 3 . In general, acridine atomic charges in 9-AA $\mathbf{1}(\mathbf{a}-\mathbf{e})$ are delocalized by resonance effects.

Significant changes in atomic charges are observed in the carbon atoms adjacent to the acridine N14 in the complexes of 9-AA 1(a-e) with DNA base-pairs. See Table 3. However the highest changes are displayed in the complexes with GC. Note, for instance, N14 in 9-AA 1(d) changes from -0.611 to 0.101 by interacting with GC while with AT it changes to -0.127 . See Table 3.

The study of the charges in the complexes of 9-AA 1(a-e) with base-pairs showed that the strongest interaction with GC is 9-AA 1(d) while the weakest is with 9-AA 1(b). Accordingly, the study of atom charges involved in hydrogen bonding in GC and respective complexes with 9-AA 1(a-e) denoted the highest charge changes in N5. Note also N5 in GC changes from -0.618 to -0.084 in the complex with 9 -AA $\mathbf{1}(\mathbf{d})$. See Table 2.

On the other hand, peptide chains in 9-AA 1(a-e) exhibit intramolecular hydrogen bonding. Significant atomic charges and torsion angles changes are also observed in the peptide chains after complex formation with base-pairs. Torsion angle changes in 9-AA 1(a) and (b) are observed from C25, causing a hindrance to intramolecular hydrogen-bonding formation. Oxygen atoms in the peptide chains of 9-AA $\mathbf{1}(\mathbf{d})$ and (e) are oriented towards hydrogen in GC causing a hindrance in the formation of intramolecular hydrogen bonding as well.

9-AA 1(a-e) and DNA base-pairs are electron-deficient aromatics, and the quadrupole moment is reversed due to the electronegativity of N14 and N15 in 9-AA and electronwithdrawing functional groups in base-pairs; see Figure 5. Face-centered stacking is disfavored in the complexes of 9-AA with base-pairs by repulsive quadrupole/quadrupole interactions. By offsetting one of the rings, a displaced configuration reduces the repulsive interactions and stabilizes complexes configuration enhancing electrostatic interactions and hydrogen bonding.

In particular 9-AA $\mathbf{1}(\mathbf{a}, \mathbf{b}$, and e) exhibit and electrostatic interaction between positively charged hydrogen and N17 in their complexes with AT, giving rise to in plane complexes. In the complex of 9-AA $\mathbf{1}(\mathbf{c})$ and $\mathrm{AT}$, repulsive interaction is overcome by hydrogen bonding between AT H30 and the carbonyl of the first Ile residue of the 9-AA. Also a hydrogen bonding between $\mathrm{H} 32$ of 9-AA 1(c) with second Ile carbonyl oxygen and another hydrogen bonding between oxygen of first Ile residue with $\mathrm{H} 30$ in AT and with H16 in 9-AA 1(c) allows the stabilization of the calculated configuration.

The complex of 9-AA $\mathbf{1}(\mathbf{d})$ with AT has shows an inverted position of the base-pair, allowing direct interaction between hydrogen joined to the most negatively charged N21 of AT 
<smiles>[2H]n1c(C)nc2c(N(C)[18F])nc(C)nc21</smiles>

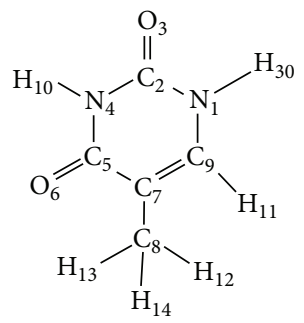<smiles>Cc1nc2c(=O)n([125I])c(N([13CH3])[13CH3])nc2n1N</smiles><smiles>CN(C)c1cc(P)n(P)c(=O)n1</smiles>

FIgURE 2: Base-pair numbering AT and GC.
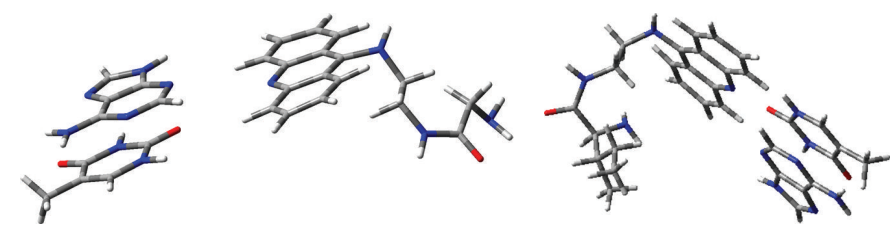

$1(\mathbf{a}) \cdots \mathrm{AT}$

$\mathbf{1}(\mathbf{b}) \cdots \mathrm{AT}$
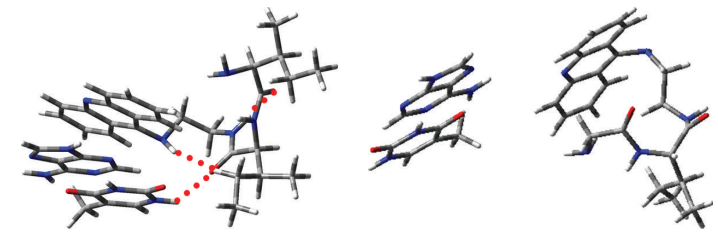

$1(\mathrm{c}) \cdots \mathrm{AT}$

$1(d) \cdots \mathrm{AT}$

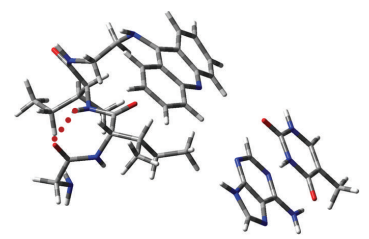

$1(\mathrm{e}) \cdots \mathrm{AT}$

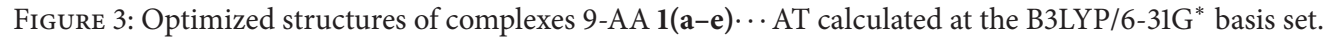




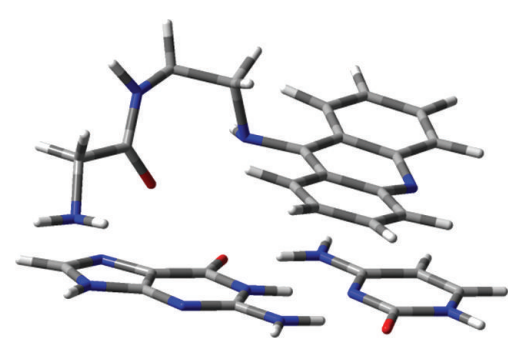

1(a) $\cdots \mathrm{GC}$

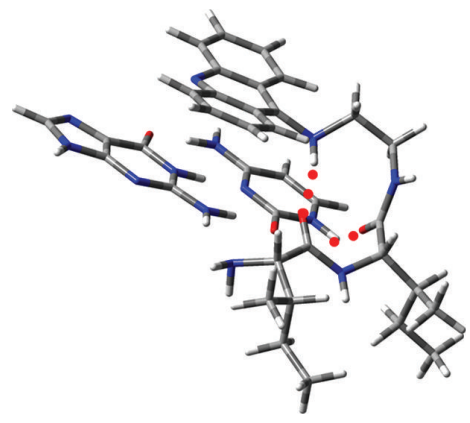

1(c) $\cdots \mathrm{GC}$

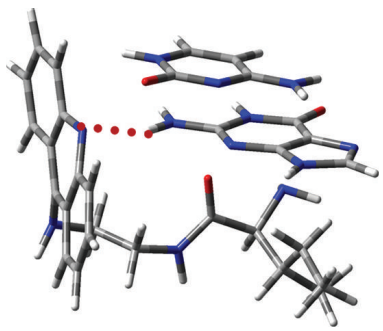

1(b) $\cdots \mathrm{GC}$

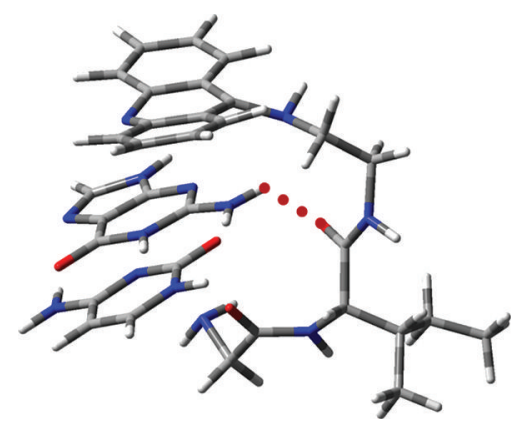

1(d) $\cdots \mathrm{GC}$

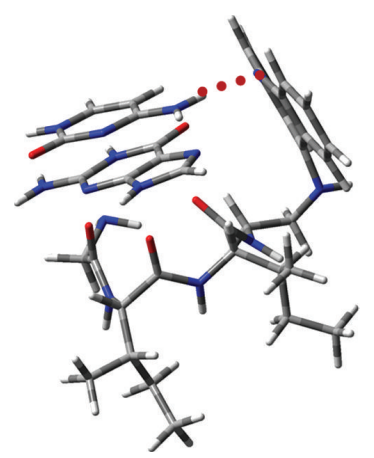

$1(e) \cdots G C$

FIGURE 4: Optimized structures of complexes 9-AA 1(a-e) $\cdots$ GC calculated at the B3LYP/6-31G* basis set.

TABLE 2: AT, GC atomic charges* and complex with 9-AA 1(a-e).

\begin{tabular}{lcccccccccc}
\hline Atom no. & AT & AT $\cdots \mathbf{1}(\mathbf{a})$ & AT $\cdots \mathbf{1}(\mathbf{b})$ & AT $\cdots \mathbf{1}(\mathbf{c})$ & AT $\cdots \mathbf{1}(\mathbf{d})$ & GC & GC $\cdots \mathbf{1}(\mathbf{a})$ & GC $\cdots \mathbf{1}(\mathbf{b})$ & GC $\cdots \mathbf{1}(\mathbf{c})$ & GC $\cdots \mathbf{1}(\mathbf{d})$ \\
\hline C20 & 0,479 & 0,607 & 0,714 & 0,375 & 0,473 & 0,554 & 0,682 & 0,577 & 0,491 \\
N15 & $-0,395$ & $-0,398$ & $-0,460$ & $-0,405$ & $-0,433$ & $-0,598$ & $-0,253$ & $-0,534$ & $-0,532$ & $-0,353$ \\
N21 & $-0,975$ & $-0,996$ & $-1,002$ & $-0,924$ & $-0,926$ & 0,500 & 0,510 & 0,518 & 0,512 \\
H26 & 0,428 & 0,432 & 0,436 & 0,422 & 0,437 & 0,742 & 0,461 & 0,479 & $-0,877$ & 0,533 \\
H27 & 0,528 & 0,528 & 0,530 & 0,534 & 0,530 & $-0,618$ & $-0,425$ & $-0,464$ & $-0,062$ & $-0,084$ \\
N4 & $-0,790$ & $-0,829$ & $-0,744$ & $-0,783$ & $-0,776$ & $-0,892$ & $-0,912$ & $-0,870$ & $-0,976$ & $-0,931$ \\
O6 & $-0,586$ & $-0,578$ & $-0,563$ & $-0,563$ & $-0,572$ & $-0,604$ & $-0,493$ & $-0,572$ & $-0,515$ & $-0,396$ \\
O3 & $-0,523$ & $-0,508$ & $-0,181$ & $-0,519$ & $-0,519$ & 0,567 & 0,573 & 0,594 & 0,566 & 0,576 \\
H25 & 0,224 & 0,235 & 0,281 & 0,235 & 0,223 & 0,517 & 0,526 & 0,519 & 0,569 & 0,542 \\
H10 & 0,528 & 0,526 & 0,525 & 0,529 & 0,529 & 0,420 & 0,421 & 0,497 & 0,461 & 0,511 \\
C2 & 0,776 & 0.719 & 0,282 & 0,765 & 0,770 & 0,411 & 0,416 & 0,407 & 0,414 & 0,416 \\
\hline
\end{tabular}

${ }^{*}$ Mulliken charges calculated at the B3LYP/6-31G* basis set. 


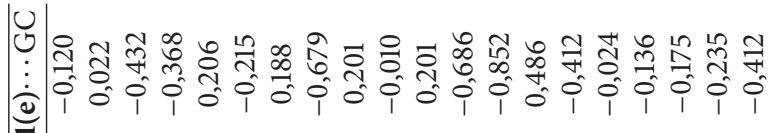

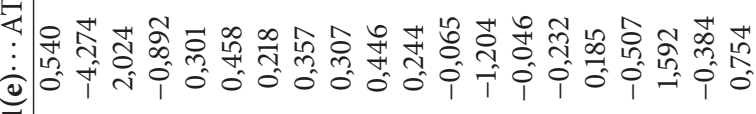

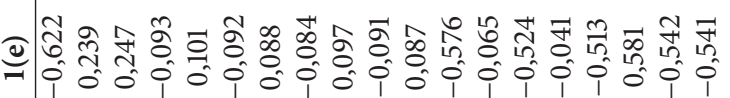

(0)

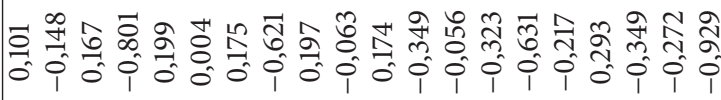

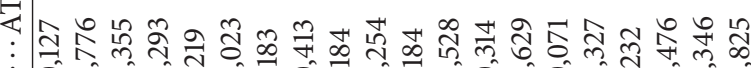

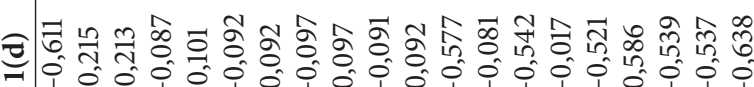

$\circ$

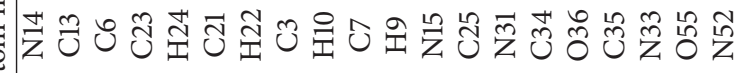

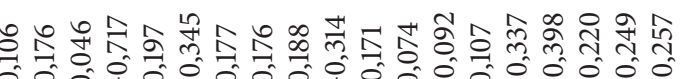

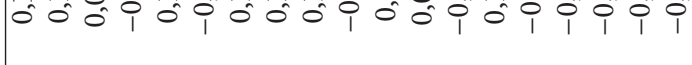

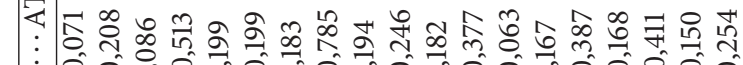

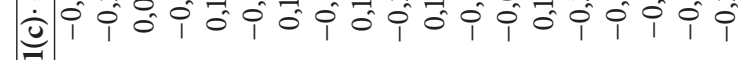

西

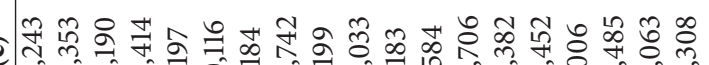

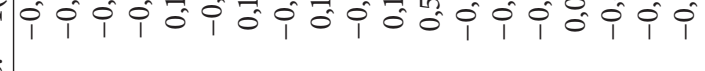

$\dot{8}$

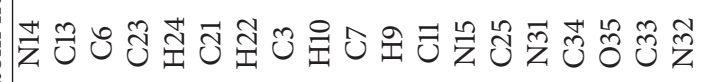

U

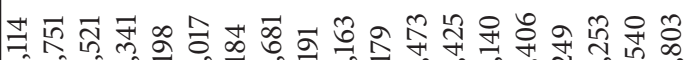

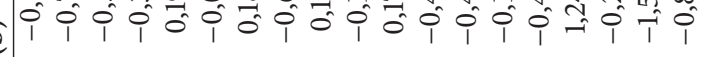

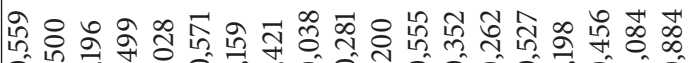

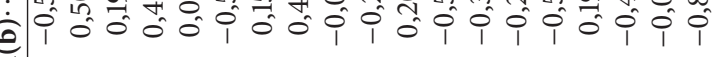

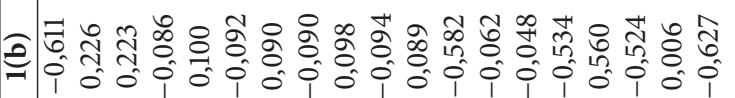

0

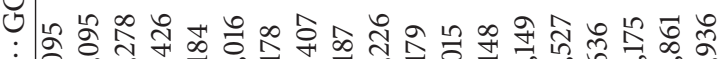

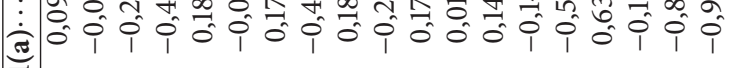

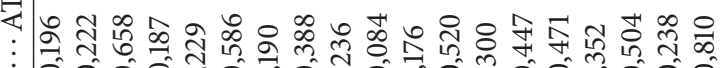
ङ

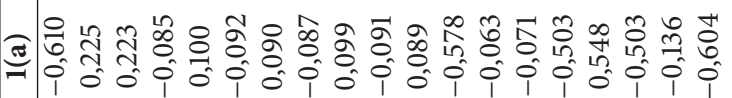

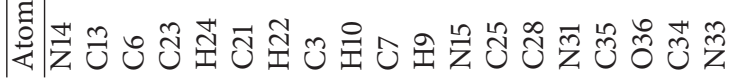


TABLE 4: HOMO and LUMO energies (ev), polarizabilities (au), and dipole moments (D) of 9-AA $\mathbf{1}(\mathbf{a}-\mathbf{e})$, base pairs and isolated bases, calculated at the B3LYP/6-31G ${ }^{* *}$ basis set.

\begin{tabular}{lccccc}
\hline Molecule & Polarizability & Dipole moment & HOMO & LUMO & $\Delta$ E (HOMO - LUMO) \\
\hline $\mathbf{1}(\mathbf{a})$ & 241,508 & 3,434 & $-0,197$ & $-0,069$ & 0,128 \\
$\mathbf{1}(\mathbf{b})$ & 202,861 & 1,643 & $-0,198$ & $-0,070$ & 0,128 \\
$\mathbf{1}(\mathbf{c})$ & 350.554 & 5,571 & $-0,212$ & $-0,083$ & 0,129 \\
$\mathbf{1}(\mathbf{d})$ & 274,299 & 3,615 & $-0,202$ & $-0,075$ & 0,127 \\
$\mathbf{1}(\mathbf{e})$ & 298,449 & 3,109 & $-0,194$ & $-0,066$ & 0,128 \\
Adenine & 109,834 & 2,366 & $-0,219$ & $-0,018$ & 0,201 \\
Guanine & 120,114 & 7,258 & $-0,201$ & $-0,003$ & 0,198 \\
Cytosine & 90,549 & 6,838 & $-0,219$ & $-0,030$ & 0,189 \\
Thymine & 95,917 & 4,027 & $-0,240$ & $-0,037$ & 0,203 \\
A-T & 221,215 & 1,301 & $-0,215$ & $-0,032$ & 0,183 \\
G-C & 228,859 & 5,446 & $-0,190$ & $-0,051$ & 0,139 \\
\hline
\end{tabular}
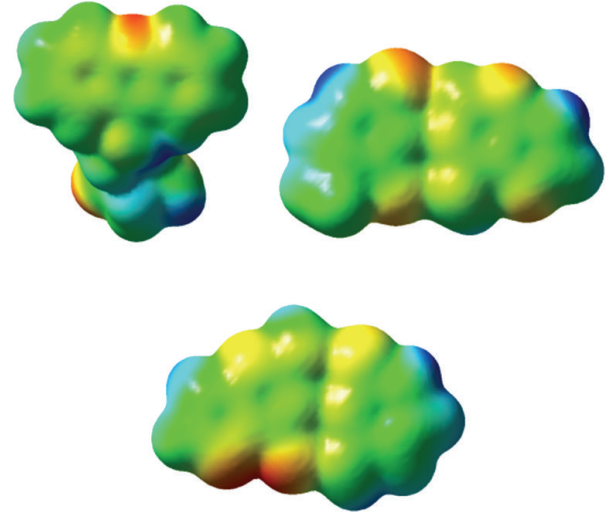

FIGURE 5: Electrostatic potential maps of 9-AA and base-pairs AT and GC, respectively.

TABLE 5: Interaction energies of optimized 9-AA 1(a-e)-base-pair complexes calculated at the B3LYP/6-31G* basis set.

\begin{tabular}{lcc}
\hline 9-AA & $\begin{array}{c}\text { AT complex } \\
\text { Interaction energy } \\
(\mathrm{kcal} / \mathrm{mol})\end{array}$ & $\begin{array}{c}\text { GC complex } \\
\text { Interaction energy } \\
(\mathrm{kcal} / \mathrm{mol})\end{array}$ \\
\hline $\mathbf{1 ( a )}$ & 2.1768 & 22.5185 \\
$\mathbf{1 ( b )}$ & 102.6410 & 26.2384 \\
$\mathbf{1 ( c )}$ & 1.2600 & 51.8498 \\
$\mathbf{1 ( d )}$ & 41.2061 & 52.4651 \\
$\mathbf{1 ( e )}$ & 42.3968 & 66.2667 \\
\hline
\end{tabular}

and N14 of 9-AA 1(d). Also both Ile carbonyl residues are directed towards the positively charged 9-AA aromatic rings.

The series of complexes with GC has shown orientation of 9-AA carbonyl oxygen of residues towards base-pair core rings as can be seen in Figure 4. Hydrogen bonding between N14 of 9-AA 1(b) and H14 in GC stabilizes the configuration. The complex between 9-AA 1(c) and GC shows 3 hydrogen bonding: intramolecular hydrogen bonding between a carbonyl oxygen of the Ile residue and H16 of 9-AA 1(c), hydrogen bonding between a carbonyl oxygen of the Ile residue and $\mathrm{H} 28$ in GC, and hydrogen bonding between 9AA 1(c) amino terminal group and H14 in GC.

Hydrogen bonding between carbonyl oxygen of Gly residue in 9-AA $\mathbf{1}(\mathbf{d})$ and $\mathrm{H} 14$ in GC stabilizes the observed configuration in Figure 4. 9-AA 1(e) shows orientation of the three carbonyl oxygen atoms of the 9-AA residues towards the base-pair ring core as stated before. This complex also has a hydrogen bonding N14 of 9-AA 1(e) and H2 of GC.

Interactions of 9-AA $\mathbf{1}(\mathbf{a}-\mathbf{e})$ with base-pairs are not based upon inherent attraction between pi cloud electron density, so pi stacking is not appropriate to describe them.

$\mathrm{C}-\mathrm{C}$ bonds in 9-AA $\mathbf{1}(\mathbf{a}-\mathbf{e})$ rings showed characteristic $\mathrm{sp}^{2}$ bond distances of polycyclic aromatic systems. 9-AA 1(ae) are rigid rings so bond distances changes are not significant by interacting with bases pairs. Base-pair bond lengths were kept almost invariable after complex interactions with 9-AA 1(a-e).

9-AA 1(a-e) have shown an asymmetric distribution of atomic charges in electrostatic potential maps; this is supported by the high dipole moments calculated; see Table 4 . Symmetric distribution of atomic charges of the polycyclic aromatic rings in 9-AA $\mathbf{1}(\mathbf{a}-\mathbf{e})$ was observed due to resonance effects.

The sequence of the intermolecular interaction energies is the same on all base-pairs: 9-AA $\mathbf{1}$ (a) $<$ 9-AA $\mathbf{1}$ (b) $<9$-AA $\mathbf{1}(\mathbf{d})<9$-AA $\mathbf{1}$ (c) $<9$-AA $\mathbf{1}$ (e) in Table 5. From the interaction energies it is seen that 9-AA $\mathbf{1}(\mathbf{c})$ and 9-AA $\mathbf{1}(\mathbf{e})$ exhibit the strongest interactions with base-pairs. These results are supported on the number of hydrogen bonding allowed for the adopted conformation in complexes of 9-AA 1(c) and 9AA 1(e).

The lowest energy difference between FMO was shown by 9 -AA $\mathbf{1}(\mathbf{d}),(0,127 \mathrm{eV})$, in Table 4 . 9-AA $\mathbf{1}(\mathbf{a}-\mathbf{e})$ showed negative energy values for LUMO and HOMO, indicating that they are good electron acceptors. LUMO energies of isolated bases and base-pairs are more positive than LUMO energies in 9-AA 1(a-e), so isolated bases and base-pairs possess electron-donating abilities, which are supported by the high ionization potential $[24,25]$. Among the isolated 
bases Guanine is the best nucleophile having the highest LUMO energy value. Thus the electron donor-electron acceptor contribution could play a role in the interacting systems.

The highest dipole moment in the 9-AA 1(a-e) series was found in 9-AA 1(c), favoring electrostatic attractions with base-pairs. As long as polarizability is in isolated bases, Guanine also exhibits the highest value (120,114 au) and GC is the most polarizable base-pair $(228,859 \mathrm{au})$; see Table 4 . 9-AA 1(a-e) have polarizabilities from 350.554 to 202.861 au, 9-AA being $\mathbf{1}(\mathbf{c})$ the largest.

Finally, high polarizability favors interactions between the systems and also high dipole moment favors electrostatic interactions. From Table 4 it can be noted that 9-AA 1(e) has a large polarizability $(298,449 \mathrm{au})$ but also has a lower dipole moment when compared to 9-AA $\mathbf{1}(\mathbf{d})$ and $\mathbf{1}(\mathbf{c})$. The strongest interactions of the complexes are then a result of mainly polarizability, hydrogen bonding, and dipole moments. The weakest interaction is found with low dipole moments and low polarizability. Charge transfer, on the other hand, could play a less significant role as there is no significant difference between FMO energies of the systems.

\section{Conclusion}

9-AA 1(a-e) have been shown to have high polarizabilities, high dipole moments, and electron acceptors abilities; on the opposite side, base-pairs are electron donors, resulting in favorable interactions between the systems under examination in this paper. From the 9-AA under consideration, the strongest interaction was exhibited by 9 -AA $\mathbf{1}(\mathrm{c})$ and 9 -AA 1(e). The weakest interaction was observed with 9-AA 1(b).

Complex interactions are stabilized by electrostatic and hydrogen-bonding contributions while charge transfer is not significant for the type of molecules under study. Theoretical procedures that cover the type of interactions described before are therefore a first good approximation towards the study of 9-aminoacridines.

\section{Acknowledgments}

The authors greatly appreciate the financial support of the Dirección de Investigación, Desarrollo e Innovación, and DIDI at the Universidad del Norte.

\section{References}

[1] P. Belmont, J. Bosson, T. Godet, and M. Tiano, "Acridine and acridone derivatives, anticancer properties and synthetic methods: where are we now?" Anti-Cancer Agents in Medicinal Chemistry, vol. 7, no. 2, pp. 139-169, 2007.

[2] F. Charmantray, M. Demeunynck, D. Carrez et al., "4Hydroxymethyl-3-aminoacridine derivatives as a new family of anticancer agents," Journal of Medicinal Chemistry, vol. 46, no. 6, pp. 967-977, 2003.

[3] R. A. Heald and M. F. G. Stevens, "Antitumour polycyclic acridines. Palladium(0) mediated syntheses of quino[4,3,2$\mathrm{kl}$ ] acridines bearing peripheral substituents as potential telomere maintenance inhibitors," Organic \& Biomolecular Chemistry, vol. 19, pp. 3377-3389, 2003.
[4] R. Hegde, P. Thimmaiah, M. C. Yerigeri, G. Krishnegowda, K. N. Thimmaiah, and P. J. Houghton, "Anti-calmodulin acridone derivatives modulate vinblastine resistance in multidrug resistant (MDR) cancer cells," European Journal of Medicinal Chemistry, vol. 39, no. 2, pp. 161-177, 2004.

[5] M. Kimura, A. Kato, and I. Okabayashi, "Acridine derivatives. IV. Synthesis, molecular structure, and antitumor activity of the novel 9-anilino-2,3-methylenedioxyacridines," Journal of Heterocyclic Chemistry, vol. 29, no. 1, pp. 73-80, 1992.

[6] M. Fujiwara, M. Okamoto, M. Okamoto et al., "Acridone derivatives are selective inhibitors of HIV-1 replication in chronically infected cells," Antiviral Research, vol. 43, no. 3, pp. 189-199, 1999.

[7] N. W. Luedtke, Q. Liu, and Y. Tor, "RNA-ligand interactions: affinity and specificity of aminoglycoside dimers and acridine conjugates to the HIV-1 Rev response element," Biochemistry, vol. 42, no. 39, pp. 11391-11403, 2003.

[8] I. B. Taraporewala, "Thiazolo[5,4- $b]$ acridines and thiazolo[4,5b]acridines: probable pharmacophores of antiviral and antitumor marine alkaloids," Tetrahedron Letters, vol. 32, no. 1, pp. 39-42, 1991.

[9] C. Korth, B. C. May, F. E. Cohen, and S. B. Prusiner, "Acridine and phenothiazine derivatives as pharmacotherapeutics for prion disease," Proceedings of the National Academy of Sciences of the United States of America, vol. 98, no. 17, pp. 9836-9841, 2001.

[10] B. C. May, A. T. Fafarman, S. B. Hong et al., "Potent inhibition of scrapie prion replication in cultured cells by bis-acridines," Proceedings of the National Academy of Sciences of the United States of America, vol. 100, no. 6, pp. 3416-3421, 2003.

[11] H. P. Kavitha, "Synthesis and antimicrobial activity of 1-(9'acridinyl)-5-(4-substituted phenyl) tetrazoles," Asian Journal of Chemistry, vol. 16, no. 2, pp. 1191-1193, 2004.

[12] S. M. Sondhi, M. Johar, N. Singh, R. Shukla, R. Raghubir, and S. G. Dastidar, "Synthesis of sulpha drug acridine derivatives and their evaluation for anti-inflammatory, analgesic and anticancer activity," Indian Journal of Chemistry B, vol. 41, no. 12, pp. 26592666, 2002.

[13] A. Albert, Ricard Clay and Company, Ltda, 1951.

[14] I. Lasnitzki and J. H. Wilkinson, "The effect of acridine derivatives on growth and mitoses of cells in vitro," British Journal of Cancer, vol. 2, pp. 369-375, 1948.

[15] J. B. Le Pecq, M. Le Bret, J. Barbet, and B. Roques, "DNA polyintercalating drugs: DNA binding of diacridine derivatives," Proceedings of the National Academy of Sciences of the United States of America, vol. 72, no. 8, pp. 2915-2919, 1975.

[16] J. Sebestik, I. Stibor, and J. Hlavcek, "New peptide conjugates with 9-aminoacridine: synthesis and binding to DNA," Journal of Peptide Science, vol. 12, no. 7, pp. 472-480, 2006.

[17] C. B. Carlson and P. A. Beal, "Point of sttachment and sequence of immobilized peptide-acridine conjugates control affinity for nucleic acids," Journal of the American Chemical Society, vol.124, no. 29 , pp. 8510-8511, 2002.

[18] J. Sponer, J. Leszczynski, and O. Hobza, "Nature of nucleic acidbase stacking: nonempirical ab initio and empirical potential characterization of 10 stacked base dimers. Comparison of stacked and H-bonded base pairs," The Journal of Physical Chemistry A, vol. 100, no. 13, pp. 5590-5596, 1996.

[19] P. Hobza, M. Kabelac, J. Sponer, P. Mejzlik, and J. Vondrasek, "Performance of empirical potentials (AMBER, CFF95, CVFF, 
CHARMM, OPLS, POLTEV), semiempirical quantum chemical methods (AM1, MNDO/M, PM3), and ab initio HartreeFock method for interaction of DNA bases: comparison with nonempirical beyond Hartree-Fock results," Journal of Computational Chemistry, vol. 18, no. 9, pp. 1136-1150, 1997.

[20] S. Riahi, M. R. Ganjali, A. B. Moghaddam, P. Norouzi, and M. Niasari, "Determination of the electrode potentials for substituted 1,2-dihydroxybenzenes in aqueous solution: theory and experiment," Journal of Molecular Structure, vol. 774, no. 13, pp. 107-111, 2006.

[21] S. Riahi, M. R. Ganjali, A. B. Moghaddam, and P. Norouzi, “Theoretical and experimental study of electrical and electrochemical properties of (E)-3-(4,5-dihydroxy-2-(phenylsulphonyl) phenyl) acrylic acid as a new caffeic acid derivative," Journal of Theoretical and Computational Chemistry, vol. 6, no. 2, pp. 255268, 2007.

[22] S. Riahi, A. B. Moghaddam, M. R. Ganjali, and P. Norouzi, "Determination of the oxidation potentials of pyrogallol and some of its derivatives: theory and experiment," Journal of Theoretical and Computational Chemistry, vol. 6, no. 2, pp. 331340, 2007.

[23] M. J. Frisch, G. W. Trucks, H. B. Schlegel et al., "Gaussian 09, Revision A02," Gaussian, Inc, Wallingford, UK, 2009.

[24] F. Prat, K. N. Houk, and C. S. Foote, "Effect of Guanine stacking on the oxidation of 8-oxoguanine in B-DNA," Journal of the American Chemical Society, vol. 120, no. 4, pp. 845-846, 1998.

[25] A. O. Colson and M. D. Sevilla, "Elucidation of primary radiation damage in DNA through application of $\mathrm{Ab}$ initio molecular orbital theory," International Journal of Radiation Biology, vol. 67, no. 6, pp. 627-645, 1995. 

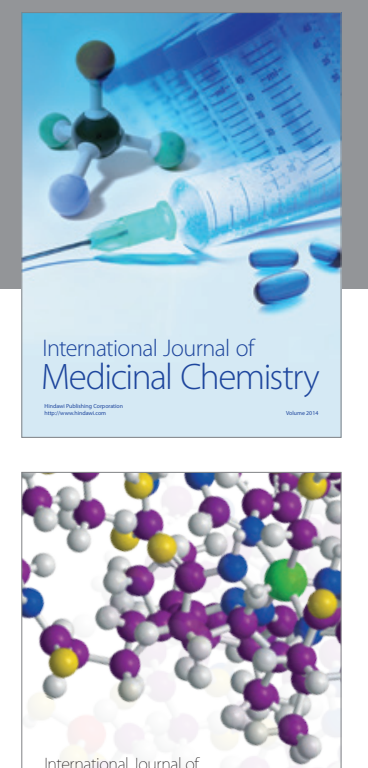

\section{Carbohydrate} Chemistry

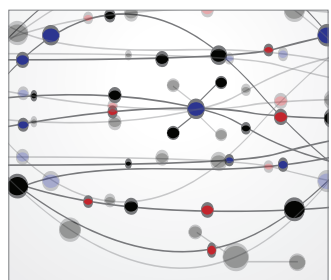

The Scientific World Journal
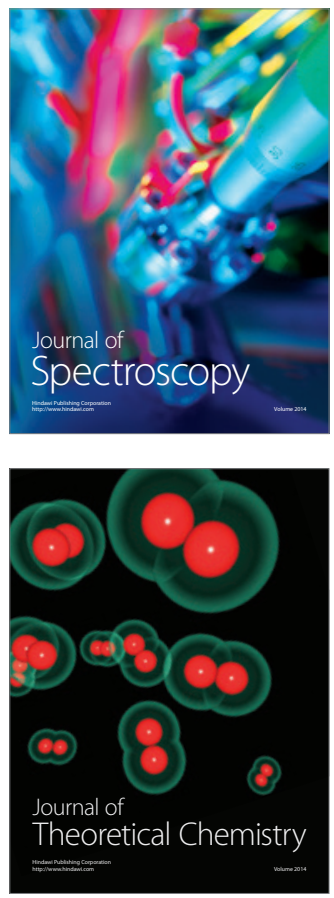
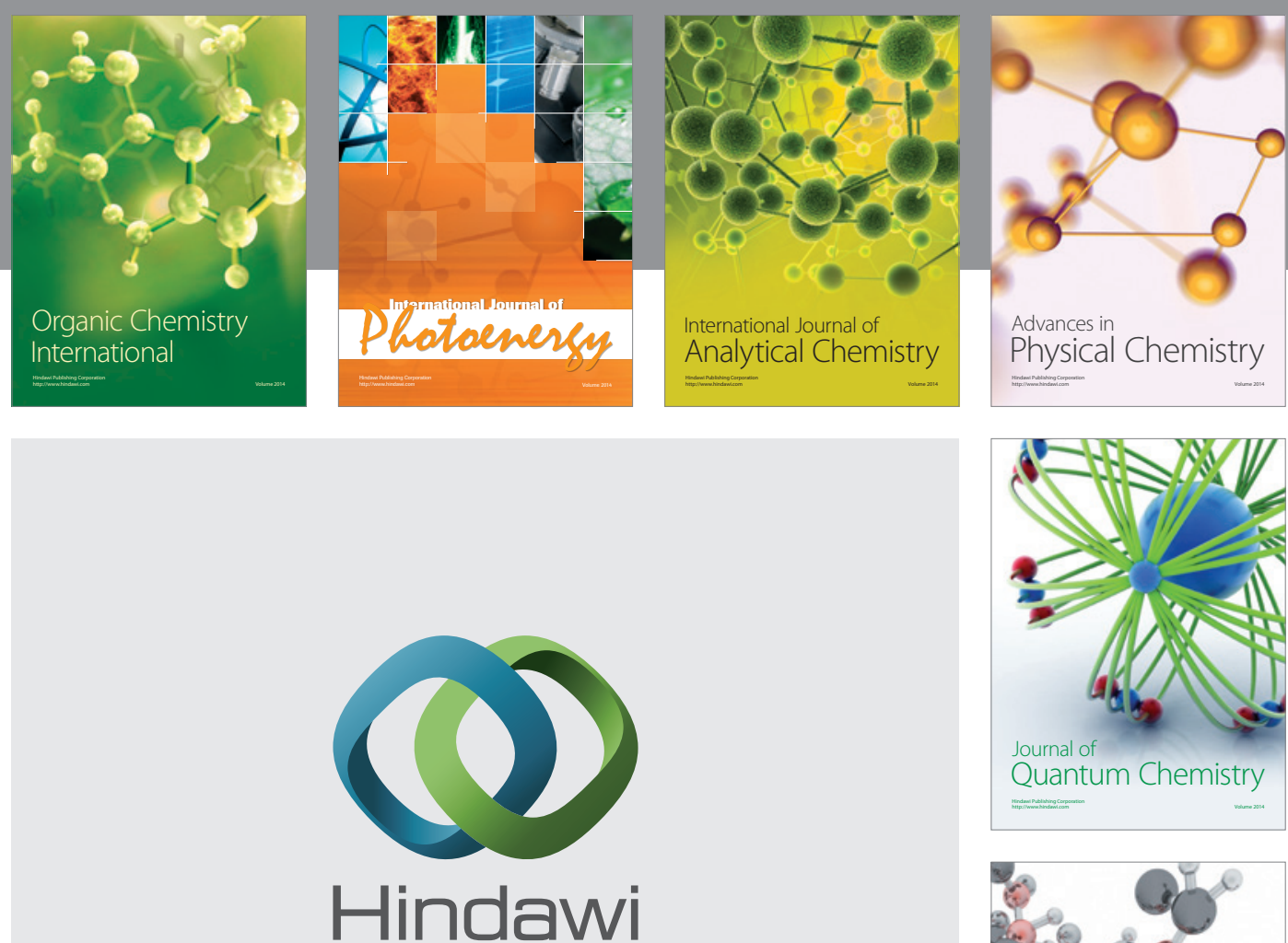

Submit your manuscripts at

http://www.hindawi.com

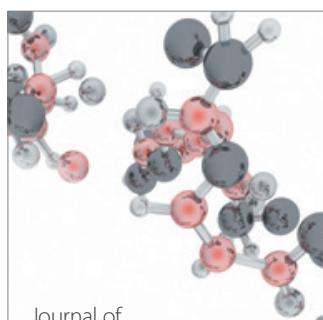

Analytical Methods

in Chemistry

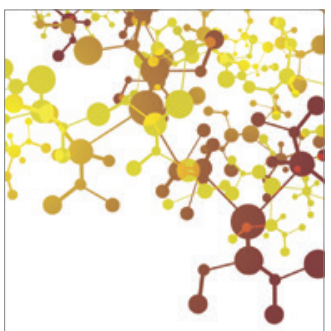

Journal of

Applied Chemistry

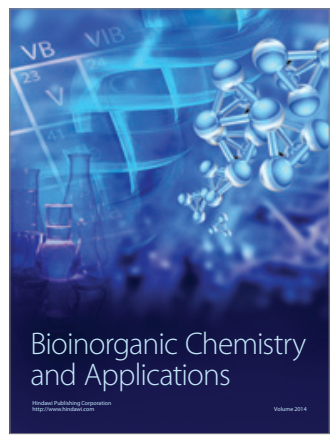

Inorganic Chemistry
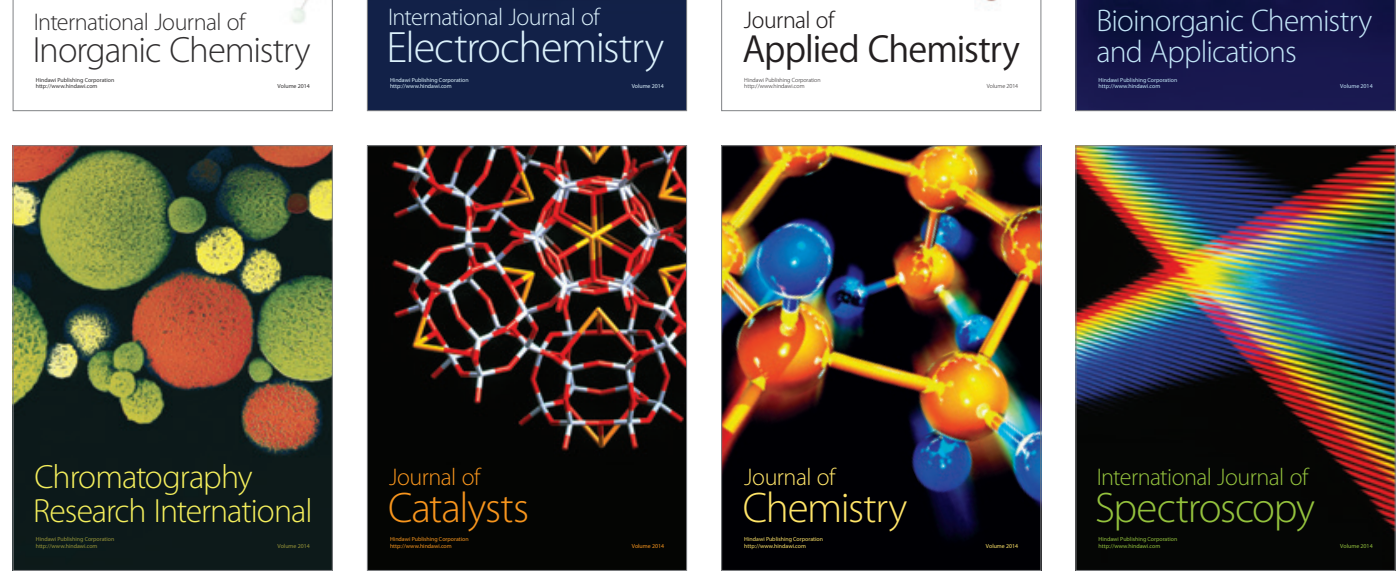